\title{
Energy-Efficiency of Distributed Antenna Systems Relying on Resource Allocation
}

\author{
Xiaoge Huang $^{{ }^{*}}$, Dongyu Zhang ${ }^{1}$, Weipeng Dai ${ }^{1}$ and She Tang ${ }^{1}$ \\ ${ }^{1}$ School of Communication and Information Engineering, \\ Chongqing University of Posts and Telecommunications \\ [E-mail : Huangxg@cqupt.edu.cn] \\ *Corresponding author : Xiaoge Huang
}

Received September 18, 2017; revised April 19, 2018; accepted September 12, 2018; published March 312019

\begin{abstract}
Recently, to satisfy mobile users' increasing data transmission requirement, energy efficiency (EE) resource allocation in distributed antenna systems (DASs) has become a hot topic. In this paper, we aim to maximize EE in DASs subject to constraints of the minimum data rate requirement and the maximum transmission power of distributed antenna units (DAUs) with different density distributions. Virtual cell is defined as DAUs selected by the same user equipment (UE) and the size of virtual cells is dependent on the number of subcarriers and the transmission power. Specifically, the selection rule of DAUs is depended on different scenarios. We develop two scenarios based on the density of DAUs, namely, the sparse scenario and the dense scenario. In the sparse scenario, each DAU can only be selected by one UE to avoid co-channel interference. In order to make the original non-convex optimization problem tractable, we transform it into an equivalent fractional programming and solve by the following two sub-problems: optimal subcarrier allocation to find suitable DAUs; optimal power allocation for each subcarrier. Moreover, in the dense scenario, we consider UEs could access the same channel and generate co-channel interference. The optimization problem could be transformed into a convex form based on interference upper bound and fractional programming. In addition, an energy-efficient DAU selection scheme based on the large scale fading is developed to maximize EE. Finally, simulation results demonstrate the effectiveness of the proposed algorithm for both sparse and dense scenarios.
\end{abstract}

Keywords: Distributed antenna systems, resource allocation, energy efficiency, virtual cell

This work is supported by the National Natural Science Foundation of China (61831002,61401053), and Innovation Project of the Common Key Technology of Chongqing Science and Technology Industry (cstc2018jcyjAX0383) 


\section{Introduction}

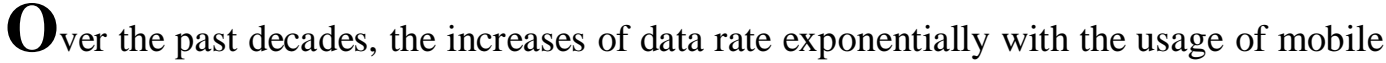
devices lead to the consumption of more energy in wireless networks [1]. Due to increasing energy consumption, greenhouse gas emissions such as carbon dioxide and methane has become great threat to the environmental protection which have increased rapidly with society development. Energy efficiency (EE) has become a key concern from the viewpoint of green communications, and considered as an important design criteria for future wireless communication systems. Besides, with the rapid development of smart devices, we need to optimize the limited resource allocation to increase the transmission data rate, as well as energy-efficiency based on new network architecture.

In recent years, DASs have played an important role in improving the quality of communication services, to provide higher system capacity, broader coverage and lower transmission power than traditional systems [2]-[3]. In a typical DAS, remote access units (RAUs) are geographically distributed in a cell and connected to the central processing unit $(\mathrm{CU})$ via optical fiber or cable. If the distance between distributed antenna units (DAUs) and mobile users is short, DASs could extend the coverage and improve EE [4].

In [5], the authors studied EE in the generalized DAS and proposed a joint subchannel and power control algorithm to maximize EE on the constraint of antenna's power budget. However, the authors did not consider users' date rate requirement. An EE optimization method was investigated in downlink multiuser OFDM-DASs in [6], the authors proposed an EE scheme by optimization jointly subcarrier assignment and power allocation based on the constraints of min-rate requirement, subcarrier reuse, and RAU's transmit power. Since the optimization function is a joint optimization problem, the authors consider it from several aspects and solved by low-complexity methods. In [7], the authors proposed an EE allocation scheme with proportional fairness in OFDM-DASs. In [8], to maximize the total transmission data rate of cognitive radio networks, the authors introduced a sensing based power allocation scheme. In [9], the authors proposed an dynamical power allocation scheme in DASs with the average transmission rate constraint and the maximum transmission power constraint, which was divided into subcarriers association and transmission power allocation optimization.

In a DAS, antenna units selection plays a key role to maximize system's EE. A proper antenna selection method can significantly improve EE. In [10], the authors proposed a joint power allocation and antenna selection algorithm to increase EE under the constraint of per RAU transmission power in OFDM- DAS. [11] - [16] also studied the solution for optimal antenna selection and power allocation to obtain maximum EE which satisfied the minimium rate constraint and antenna association constraint.

In this paper, we study energy-efficiency of DASs relying on optimal resource allocation, with different DAU density: sparse and dense. Virtual cell is defined as DAUs selected by the same UE which related with the number of subcarriers and each DAU's transmission power. In the sparse DAS scenario, we maximize EE under the constrain of 
transmission power and DAU selection rules. Because of the optimization function is a joint optimization problem, we divide it into the following two sub-problems: joint DAU selection with subcarrier allocation algorithm (JDSA) and optimal power allocation algorithm to achieve optimal results. Different from the sparse DAS scenario, user could access the same channel in dense DAS scenario, thus co-channel interference should be taken into consideration. According to the characteristics of the original problem, the interference upper bound and the fractional programming theory is used to solve the problem.

The rest of the paper is organized as follows: the system model and the problem formulation is presented in Section 2. We introduce energy-efficient of DASs resource allocation schemes in the sparse and the dense DAS scenario respectively in Section 3. In Section 4, simulation results and performance valuation are discussed. At last, we make a short summary conclusion in Section 5.

\section{System Model and Problem Formulation}

\subsection{System Model}

Consider a downlink multiuser single-cell Orthogonal Frequency Division Multiplexing distributed antenna system (OFDM-DAS), with the cell radius $R, M$ DAUs, $K$ UEs, $M \geq K$, and $N$ subcarriers, as shown in Fig. 1 .

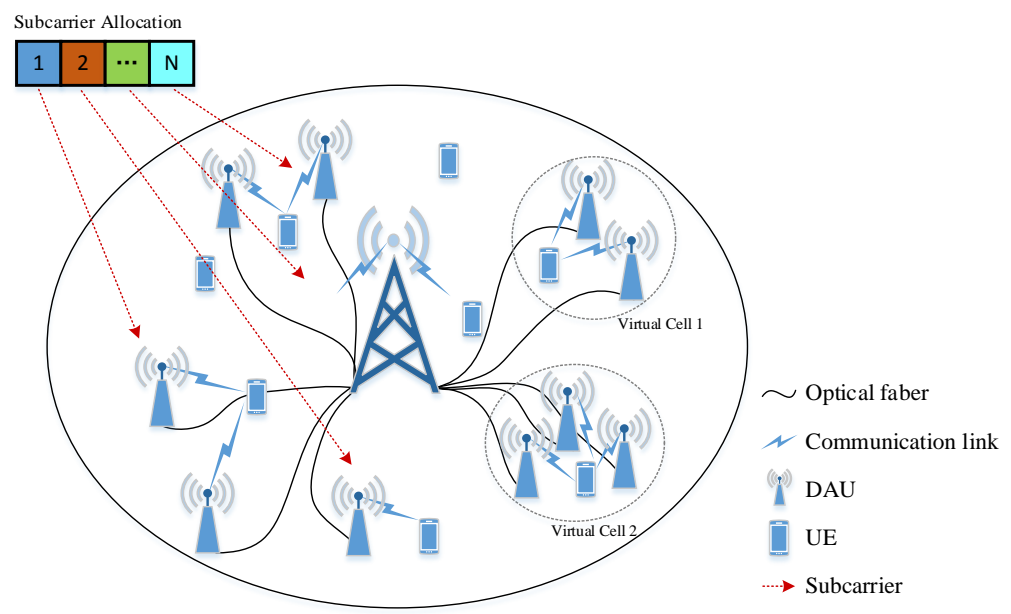

Fig. 1. System model

Let $W$ denote the total bandwidth, which is divided into $N$ unit parts. Assume that the channel state information (CSI) is available and the signal to noise ratio (SNR) of user $k$ on subcarrier $n$ from DAU $m$ is given by: 


$$
\gamma_{k, n, m}=\frac{p_{k, n, m}\left|h_{k, n, m}\right|^{2}}{\sigma^{2}}
$$

where $p_{k, n, m}$ is the transmission power from DAU $m$ to user $k$ on subcarrier $n$, $h_{k, n, m}$ is the channel impulse response between DAU $m$ and user $k$ on subcarrier $n$, which includes the small-scale and the large-scale fading. $h_{k, n, m}=l_{k, m} \cdot g_{k, n, m}$, where the large scale fading factor is denoted as $l_{k, m}=\sqrt{s_{k, m} \cdot d_{k, m}^{-\alpha}}, s_{k, m}$ is the shadowing variable, $d_{k, m}$ represents the distance between DAU $m$ and UE $k$ with the path-loss index $\alpha . g_{k, n, m}$ is the small-scale fading factor which is a distributed complex Gaussian random variable with zero variance and zero mean. Therefore, the transmission data rate of UE $k$ is calculated by:

$$
r_{k}=B \sum_{m=1}^{M} \sum_{n=1}^{N} a_{k, m} b_{n, m} \log _{2}\left(1+\gamma_{k, n, m}\right)
$$

where $a_{k, m}=[0,1]$ indicates the association of DAU $m$ and user $k$, and $b_{n, m}=[0,1]$ denotes the association of subcarrier $n$ and DAU $m$. Hence, the total transmission data rate of DASs can be written as:

$$
R_{\text {tot }}=\sum_{k=1}^{K} r_{k}
$$

In this paper, EE of DASs is defined as the ratio of total rate to total power consumption. The total power consumption is composed by the dynamic power consumption and the static power consumption. The first part comes from the real-time transmission power consumption, whereas the second part comes from the circuit power consumption and backhaul power consumption caused by fiber-transmission. Therefore, Equation 4 below shows the characteristics of the model:

$$
P_{t o t}=\frac{P_{t}}{\tau}+M p_{c}+p_{b h}
$$

where $P_{t}=\sum_{k=1}^{K} \sum_{m=1}^{M} \sum_{n=1}^{N} a_{k, m} b_{n, m} p_{k, n, m}$ is the total transmit power and $\tau$ is the power amplifier efficiency. $p_{c}$ is the circuit power consumption. $p_{b h}$ is the backhaul power consumption. Thus, EE of DASs can be expressed as:

$$
\eta_{E E}=\frac{R_{\text {tot }}}{P_{\text {tot }}}
$$




\subsection{Problem Formulation}

\section{Sparse DAS Scenario}

In the sparse DAS scenario, there is a small number of DAUs and all DAUs should be selected by UEs. Considering min-rate requirement of UEs and maximum DAU's transmission power, the optimization function of EE is given as P1:

$$
\begin{aligned}
\mathbf{P 1}: \max _{\boldsymbol{a}, \boldsymbol{b}, \boldsymbol{P}} E E & =\frac{R_{\text {tot }}(\boldsymbol{a}, \boldsymbol{b}, \boldsymbol{P})}{P_{\text {tot }}(\boldsymbol{a}, \boldsymbol{b}, \boldsymbol{P})} \\
\text { s.t. } C 1: r_{k} \geq r_{k}^{\text {req }}, \forall k & \\
C 2 & : \sum_{n=1}^{N} a_{k, m} b_{n, m} p_{k, n, m} \leq P_{m}^{\max }, \forall k, m \\
C 3 & : 1 \leq \sum_{m=1}^{M} a_{k, m} \leq M-K+1, \forall k \\
C 4 & : \sum_{k=1}^{K} a_{k, m} \leq 1, \forall m ; \sum_{m=1}^{M} b_{n, m} \leq 1, \forall n \\
C 5 & : a_{k, m} \in\{0,1\}, b_{n, m} \in\{0,1\}, \forall k, n, m \\
C 6 & : \sum_{n=1}^{N} b_{n, m} \leq N_{m}, \forall m \\
C 7 & : p_{k, n, m} \geq 0, \forall k, n, m
\end{aligned}
$$

where $P_{m}^{\max }$ is the maximum transmission power of DAU $m$. The constraint $C 1$ is the minimum rate requirement constraint with the minimum data rate requirement $r_{k}^{\text {req }}$. The constraint $C 2$ ensures the total transmission power budget of each DAU. The constraint C3 guarantees that at least one DAU could provide service to one user. C4 and C5 show that each DAU can be allocated to only one user, and a subcarrier could be distributed to only one DAU at a time. C6 shows the maximum available number of subcarriers for DAU. It's worth nothing that parameters $r_{k}^{\text {req }}$ and $P_{m}^{\max }$ should be set properly to make $\mathbf{P 1}$ feasible.

\section{Dense DAS Scenario}

In the dense DAS scenario, there is a large number of DAUs, since DAUs are allocated in different geographical positions, the distances between DAUs and users are different. According to the minimum data rate constraint, only a part of DAUs will be selected. Furthermore, the number of DAUs is much larger than available channels. We assume UEs could access the same channel in the dense scenario, thus co-channel interference should be taken into account. Then, the transmission rate of UE $k$ with unit bandwidth is: 


$$
r_{k}=\log _{2}\left(1+\frac{\sum_{m \in V_{k}} p_{m, k}\left|h_{m, k}\right|^{2}}{\sum_{j \neq k} \sum_{l \in V_{j}(k)} p_{l, j}\left|h_{l, k}\right|^{2}+\sigma^{2}}\right)
$$

where $p_{m, k}$ is the transmission power of DAU $m$ to user $k, V_{k}$ indicates the set of DAUs serving user $k$, namely, virtual cell $V_{k}$, and $\left|V_{k}\right|$ is the number of DAUs serving for user $k$, thus the size of the virtual cell $\sum_{j \neq k} \sum_{l \in V_{j}(k)} p_{l, j}\left|h_{l, k}\right|^{2}$ is the co-channel interference, virtual cell $V_{j}(k)$ is the set of DAUs serving user $j$ which would generate co-channel interference to UE $k$. The overall power consumption in the dense DAS scenario is given by:

$$
P_{\text {tot }}=\frac{\sum_{k=1}^{K} \sum_{m \in V_{k}} p_{m, k}}{\tau}+M_{a c} p_{c}+p_{b h}
$$

where $M_{a c}$ is the number of selected DAUs. Therefore, the EE optimization problem is defined as P2:

$$
\begin{gathered}
\text { P2: } \max _{p_{m, k} \in \boldsymbol{P}} E E=\frac{R_{\text {tot }}(\boldsymbol{P})}{P_{t o t}(\boldsymbol{P})} \\
\text { s.t. } C 1: \sum_{k \in U_{m}} p_{m, k} \leq P_{m}^{\max }, \forall k \\
C 2: r_{k} \geq r_{k}^{r e q}, \forall k \\
C 3: p_{m, k} \geq 0, \forall m, k
\end{gathered}
$$

where $\boldsymbol{P}=\left[p_{m, k}\right]_{M \times K}$ is a $M \times K$ power allocation matrix, $U_{m}$ is the set of users served by DAU $m, P_{m}^{\max }$ is the maximum transmit power of DAU $m$.

\section{Energy Efficiency Resource Allocation Schemes}

\subsection{Energy Efficiency Resource Allocation of Sparse DAS}

Due to the non-convex characteristic of the optimal function P1, to obtain the optimal solution is very complicated. In this section, we introduce a low-complexity suboptimal algorithm. The original optimization problem algorithm is considered from two aspects: subcarrier allocation algorithm which could choose the relevant DAU and power allocation algorithm which could achieve the optimal power allocation. 


\section{1. subcarrier allocation algorithm}

(1) The number of subcarriers of each DAU

Generally, when the user is getting closer to a DAU, the channel conditions will become better. UE has larger probability to select the DAU which has better contribution to the data rate. SNR of user $k$ from DAU $m$ on subcarrier $n$ with unit transmission power is given as $s n r_{k, n, m}=\left|h_{k, n, m}\right|^{2} / \sigma^{2}$. Similarly, we could construct a three dimensional $M \times N \times K$ matrix, denoted as $\operatorname{SNR}_{k, n, m}(K, N, M)$, which can be represented by:

$$
\operatorname{SNR}_{k, m}(n)=\left(\begin{array}{cccc}
s n r_{1, n, 1} & s n r_{2, n, 1} & \ldots & s n r_{K, n, 1} \\
s n r_{1, n, 2} & s n r_{2, n, 2} & \ldots & s n r_{K, n, 2} \\
\vdots & \vdots & \ddots & \vdots \\
s n r_{1, n, M} & s n r_{2, n, M} & \cdots & s n r_{K, n, M}
\end{array}\right)
$$

Subsequently, the $M \times M$ matrix is summed with each row to obtain a new $1 \times M$ matrix, given by:

$$
\operatorname{SNR}_{m}(n)=\left[\sum_{k=1}^{K} s n r_{k, n, 1}, \sum_{k=1}^{K} s n r_{k, n, 2}, \cdots, \sum_{k=1}^{K} s n r_{k, n, M}\right]^{T}
$$

Let $\mathcal{P}_{m}^{n}$ denote the access probability on subcarrier $n$ of DAU $m$, expressed as:

$$
\mathcal{P}_{m}^{n}=\frac{\sum_{k=1}^{K} s n r_{k, n, m}}{\sum_{m=1}^{M} \sum_{k=1}^{K} s n r_{k, n, m}}
$$

The access probability of DAUs on subcarrier $n$ is a vector, which can be expressed by $\mathcal{P}^{n}=\left[\mathcal{P}_{1}^{n}, \mathcal{P}_{2}^{n}, \cdots, \mathcal{P}_{M}^{n}\right]^{T}$. The total access probability of DAU $m$ on all subcarriers is $\mathcal{P}_{m}=\sum_{n=1}^{N} \mathcal{P}_{m}^{n}=\mathcal{P}_{m}^{n}+\mathcal{P}_{m}^{n}+\cdots+\mathcal{P}_{m}^{N}$. Thus, we could obtain the ratio of the access probability of DAU $m$ over all DAUs, which is denoted as $\phi_{m}=\frac{\sum_{n=1}^{N} p_{m}^{n}}{\sum_{m=1}^{M} \sum_{n=1}^{N} p_{m}^{n}}$. Therefore, the maximum number of subcarriers which is assigned to DAU $m$ is $N_{m}=\left\lfloor\phi_{m} \cdot N\right\rfloor$, where $\lfloor x\rfloor$ is the floor function of $x$.

\section{(2) Optimal DAU selection with subcarrier allocation}

In order to ensure every UE can be served by at least one DAU with one subcarrier, we firstly assign DAU and subcarrier to each user based on the CSI, and the rest of the unallocated subcarriers and DAUs will be assigned based on the CSI under the constraint of the maximum available number of each DAU. The brief procedure of the algorithm is follows: 
Step 1: Sort the DAU in descend order according to CSI, find maximum $h_{k, n, m}$, and allocate the optimal DAU and the subcarrier to the related UE. Then, remove the DAU and the subcarrier in the matrix. Repeat until all UEs select a DAU.

Step 2: Assign the remaining DAUs to UEs according to CSI. Repeat until the DAUs set is empty.

Step 3: Assign the remaining subcarriers to DAUs according to CSI until the number of subcarriers of $\mathrm{DAU}_{\mathrm{m}}$ is $N_{m}$.

Table 1 gives the detail procedure of the proposed algorithm.

Table 1. Joint DAU Selection with Subcarrier Allocation Algorithm (JDSA)

\section{Joint DAU Selection with Subcarrier Allocation Algorithm (JDSA)}

\section{Initialization}

2. Step 1: Create $H(K, N, M)$

1) Choose $h_{k^{*}, n^{*}, m^{*}} \in H$; let $\Omega_{m^{*}}=\Omega_{m^{*}}+\left\{n^{*}\right\}, S_{k^{*}}=S_{k^{*}}+\left\{m^{*}\right\}$;

2) $\bar{M}=\bar{M}-m^{*}, \bar{K}=\bar{K}-k^{*}, \bar{N}=\bar{N}-n^{*}$;

3) Remove $H\left(:,:, m^{*}\right), H\left(k^{*},:,:\right)$ and $H\left(:, n^{*},:\right)$.

4) Repeat 2.1 until $|\bar{M}|_{r}=M-K,|\bar{N}|_{r}=N-K, i=0$.

3. Step 2: Construct $H^{\prime}(K, N-K, M-K)$ from Step 1

1) Choose $h_{k^{*}, n^{*}, m^{*}} \in H^{\prime}(K, N-K, M-K)$, let $m^{*} \in S_{k^{*}}, n^{*} \in \Omega_{m^{*}}$;

2) $\Omega_{m^{*}}=\Omega_{m^{*}}+\left\{n^{*}\right\} ; \bar{M}=M-K-\left\{m^{*}\right\}, \bar{N}=N-K-\left\{n^{*}\right\}$;

3) Remove $H^{\prime}\left(:,:, m^{*}\right)$ and $H^{\prime}\left(:, n^{*},:\right)$

4) Repeat 3.1 until $|\bar{M}|_{r}=0 ;|\bar{N}|_{r}=N-M ;\left|\Omega_{m}\right|=1$

4. Step 3: Get $S_{k} \neq \varnothing$, and $\sum_{k=1}^{K}\left|S_{k}\right|=M, \sum_{m=1}^{M} \Omega_{m}=M$;

1) Construct $\overline{H_{k}}\left(k, N-M,\left|S_{k}\right|\right)$;

2) Choose $h_{k^{*}, n^{*}, m^{*}} \in \bar{H}$; Let $n^{*} \in \Omega_{m^{*}}$

3) Remove $H_{k}\left(k, n^{*},:\right)$

4) Until $\left|\Omega_{m}\right|=N_{m}$

\section{Optimal Power Allocation}

According to allocation results from the previous step, the virtual cell of user $k$ is determined. Thus, equation (2) and (4) could be replaced by (13) and (14), separately:

$$
r_{k}=B \sum_{m \in S_{k}} \sum_{n \in \Omega_{m}} \log _{2}\left(1+\gamma_{k, n, m}\right)
$$




$$
P_{\text {tot }}=\sum_{k=1}^{K} \sum_{m \in S_{k}} \sum_{n \in \Omega_{m}} \frac{1}{\tau} p_{k, n, m}+M p_{c}+p_{b h}
$$

Therefore, the previous optimization function $\mathbf{P 1}$ could be transformed into optimization function P3:

$$
\begin{array}{rl}
\text { P3: } \max _{\boldsymbol{P}} & E E=\frac{R_{\text {tot }}(\boldsymbol{P})}{P_{\text {tot }}(\boldsymbol{P})} \\
\text { s.t. } C 1: r_{k} \geq r_{k}^{r e q}, \forall k \\
C 2: \sum_{n \in \Omega_{m}} p_{k, n, m} \leq P_{m}^{\mathrm{max}}, \forall k, m \\
C 7: p_{k, n, m} \geq 0, \forall k, n, m
\end{array}
$$

where $P_{\text {tot }}(P)$ and $R_{\text {tot }}(P)$ are functions of $P$. The optimization problem is convex, and the Lagrangian dual method [17] could be used to solve the problem P3. Firstly, we convert the fractional form of problem P3 into the subtraction, as shown by the formula P4:

$$
\begin{gathered}
\max _{\boldsymbol{P}} U(q)=R_{\text {tot }}(\boldsymbol{P})-q \cdot P_{\text {tot }}(\boldsymbol{P}) \\
\text { s.t. } C 1, C 2, C 7
\end{gathered}
$$

Theorem 1 We have $q^{*}=\max R_{\text {tot }}\left(\boldsymbol{P}^{*}\right) / P_{\text {tot }}\left(\boldsymbol{P}^{*}\right)$, if and only if $U\left(q^{*}\right)=R_{\text {tot }}\left(\boldsymbol{P}^{*}\right)-q^{*} \cdot P_{\text {tot }}\left(\boldsymbol{P}^{*}\right)=0$.

In Theorem 1, $\boldsymbol{P}^{*}$ is the optimal transmission power of a DAU, $q^{*}$ is the optimal EE of the DAU. Hence, the optimization problem (16) can be represented by the equivalent Lagrangian function:

$$
\begin{aligned}
& L(\boldsymbol{P}, \boldsymbol{\alpha}, \boldsymbol{\beta})=B \sum_{k=1}^{K} \sum_{m \in S_{k}} \sum_{n \in \Omega_{m}} \log _{2}\left(1+\frac{p_{k, n, m}\left|h_{k, n, m}\right|^{2}}{\sigma^{2}}\right)-\sum_{k=1}^{K} \alpha_{k}\left(r_{k}^{r e q}-r_{k}\right) \\
& -q\left(\sum_{k=1}^{K} \sum_{m \in S_{k}} \sum_{n \in \Omega_{m}} \frac{1}{\tau} p_{k, n, m}+M p_{c}+p_{b h}\right)-\sum_{m=1}^{M} \beta_{m}\left(\sum_{n \in \Omega_{m}} p_{k, n, m}-P_{m}^{\max }\right)
\end{aligned}
$$

where $\boldsymbol{\alpha}=\left[\alpha_{1}, \alpha_{2}, \cdots, \alpha_{K}\right]^{T}$ and $\boldsymbol{\beta}=\left[\beta_{1}, \beta_{2}, \cdots, \beta_{M}\right]^{T}$ are Lagrangian multipliers which are non-negative. By using the derivative with respect to $p_{k, n, m}$. We can obtain the optimal $p_{k, n, m}^{*}$, which is given as:

$$
p_{k, n, m}^{*}=\left[\frac{\left(1+\alpha_{k}\right) B}{\left(q \tau+\beta_{m}\right) \ln 2}-\frac{\sigma^{2}}{\left|h_{k, n, m}\right|^{2}}\right]^{+}
$$

In the following, we consider the water filling algorithm to solve the power allocation problem by using the sub-gradient method, which is shown as: 


$$
\begin{gathered}
\Delta \alpha_{k}^{(i)}=B \sum_{m \in S_{k}} \sum_{n \in \Omega_{m}} \log _{2}\left(1+\frac{p_{k, n, m}^{*(i)}\left|h_{k, n, m}\right|^{2}}{\sigma_{z}^{2}}\right)-r_{k}^{r e q} \\
\Delta \beta_{m}^{(i)}=P_{m}^{\max }-\sum_{n \in \Omega_{m}} p_{k, n, m}^{*(i)}
\end{gathered}
$$

where the optimal transmission power in ith iteration is $p_{k, n, m}^{*(i)}$. The update process of $\alpha_{k}$ and $\beta_{m}$ are outlined by the following equations, respectively:

$$
\alpha_{k}^{(i+1)}=\left[\alpha_{k}^{(i)}-\delta^{(i)} \Delta \alpha_{k}^{(i)}\right]^{+}, \beta_{m}^{(i+1)}=\left[\beta_{m}^{(i)}-\xi^{(i)} \Delta \beta_{m}^{(i)}\right]^{+}
$$

where $i$ is iteration index, $\delta^{(i)}$ and $\xi^{(i)}$ are iteration steps.

Based on the above analysis, Table 2 shows the EE-based optimal power allocation algorithm.

Table 2. EE-based optimal power allocation algorithm

\section{Energy Efficient-based Optimal Power Allocation Algorithm}

\section{Initialization:}

$$
\alpha, \beta, \varepsilon, \kappa, t=0, q^{(0)}=0, i=0 .
$$

\section{Repeat}

3. Calculate $p_{k, n, m}^{*(i)}$ by equation (18)

4. Repeat

5. Calculate $\alpha, \beta$ by equation (21)

6. if $\left|\alpha_{k}^{(i+1)}-\alpha_{k}^{(i)}\right| \leq \kappa,\left|\beta_{m}^{(i+1)}-\beta_{m}^{(i)}\right| \leq \kappa$

7. break

8. end if

9. Until $i \geq I_{\max }$

10. if $\left|R_{\text {tot }}\left(\boldsymbol{P}^{*}\right)-q^{(i)} P_{\text {tot }}\left(\boldsymbol{P}^{*}\right)\right| \leq \varepsilon$ or $i \geq I_{\max }$

11. $E E^{o p t}=q^{(t)}=R_{\text {tot }}\left(\boldsymbol{P}^{*}\right) / P_{\text {tot }}\left(\boldsymbol{P}^{*}\right)$.

12. break

13. else

14. Set $q^{(i+1)}=R_{\text {tot }}\left(\boldsymbol{P}^{*}\right) / P_{\text {tot }}\left(\boldsymbol{P}^{*}\right), i=i+1$

15. end if

16. Until $i \geq I_{\max }$

\subsection{Energy Efficiency Resource Allocation of the Dense DAS}

In the dense DAS scenario, UEs could select DAUs which provide better channel condition and satisfy the transmission requirement. Furthermore, the number of DAUs is 
larger than available channels. We assume UEs could share the same channel and co-channel interference should be taken into consideration. For the non-convex optimization problem P2, we propose an equivalent sub-optimal algorithm. Firstly, we obtain the virtual cell of UEs based on the large scale fading. Secondly, since the optimization problem is a non-convex problem, we relax the optimization problem to the subtractive form and solved by the bisection method in an iterative manner. Finally, we adopt a EE power distribution procedure to achieve the optimal EE which not only satisfies the transmission data rate constraint but also meets the transmission power constraint of DAUs.

\section{Optimal DAU selection algorithm based on large scale fading}

Assuming that each DAU delivers pilot signal with constant power on the dedicated control channel, every user can obtain the information of adjacent DAUs via listening the channel. let $A_{k}$ denote the set of DAUs around user $k$. CU manages the DAU selection algorithm based on the CSI from users. The DAUs which have more contribution to data rate will be selected and denoted as $V_{k}, V_{k} \in A_{k}$ is the virtual cell of user $k$.

The optimal DAU selection algorithm based on the large scale fading is carried out as follows: Firstly, calculate the large scale fading information $l_{m, k}$ between DAUs and user $k$ in $A_{k}$, then list $l_{m, k}$ in an ascending order, and select the first $\left|V_{k}\right|$ DAUs as the associate set of user $k$, namely, virtual cell of user $k$. The details of the optimal DAU selection algorithm is outlined in Table 3.

Table 3. Optimal DAU Selection Algorithm

\section{Optimal DAU Selection Algorithm}

\section{Initialization:}

$V_{k}=\varnothing, \forall k \in\{1,2, \cdots, K\}$, Set $V,|V|$ is the size of virtual cell.

2. for $k=1: K$

3. Obtain $A_{k}$ of user $k$ through listening channel

4. Caculate the large scale fading $l_{m, k}$ between DAU $m$ and user $k, m \in A_{k}$.

5. Sort all the large scale fading in ascending order, $l_{m_{1}, k}<l_{m_{2}, k}<\cdots<l_{m_{\left|k_{k}\right|}, k}$

6. Select the first $\left|V_{k}\right|$ DAUs as the virtual cell of user $k$

7. $\left|V_{k}\right|=V$ and $\left|V_{k}\right|<\left|A_{k}\right|$

8. end for

\section{EE Optimization Algorithm}

The objective function of optimization problem $\mathbf{P 2}$ is non-convex, which leads to a NP-hard nonconvex optimal function [18]. To reduce the complexity of problem P2, we 
set a threshold of the noise-plus-interference power, expressed as $I_{u}$. Thus, the expression of the approximate data rate function can be derived as:

$$
\widetilde{r_{k}}=\log _{2}\left(1+\frac{\sum_{m \in V_{k}} p_{m, k}\left|h_{m, k}\right|^{2}}{I_{u}}\right) \leq r_{k}
$$

The approximate total data rate of DASs is given as:

$$
R_{\text {tot }}=\sum_{k=1}^{K} \tilde{r}_{k}
$$

Therefore, the optimal function $\mathbf{P} 2$ could be transferred to:

$$
\begin{aligned}
& \max _{p_{m, k} \in P} \frac{\sum_{k=1}^{K} \tilde{r}_{k}\left(p_{m, k}\right)}{\sum_{k=1}^{K} \sum_{m \in V_{k}} \frac{1}{\tau} p_{m, k}+M_{a c} p_{c}+p_{b h}} \\
& \text { s.t. } C 1: \sum_{k \in U_{m}} p_{m, k} \leq P_{m}^{\max }, \forall k \\
& \quad C 2: \widetilde{r}_{k} \geq r_{k}^{r e q}, \forall k \\
& \quad C 3: p_{m, k} \geq 0, \forall m, k
\end{aligned}
$$

Based on the approximate data rate $\widetilde{r}_{k}$, the optimization problem $\mathbf{P} 2$ can be transformed into a convex optimization problem $\mathbf{P 5}$ by fractional programming, which is given as:

$$
\begin{gathered}
\text { P5: } \max _{p_{m, k} \in \boldsymbol{P}} F(\eta, p)=\sum_{k=1}^{K} \widetilde{r}_{k}\left(p_{m, k}\right)-\eta \cdot\left(\sum_{k=1}^{K} \sum_{m \in V_{k}} \frac{1}{\tau} p_{m, k}+M_{a c} p_{c}+p_{b h}\right) \\
\text { s.t. } C 1: \sum_{k \in U_{m}} p_{m, k} \leq P_{m}^{\max }, \forall k \\
C 2: \widetilde{r}_{k} \geq r_{k}^{r e q}, \forall k \\
\text { C3: } p_{m, k} \geq 0, \forall m, k
\end{gathered}
$$

According to the Theorem 1, we have:

$$
F\left(\eta^{*}, p^{*}\right)=R_{\text {tot }}\left(\boldsymbol{P}^{*}\right)-\eta^{*} \cdot P_{t o t}\left(\boldsymbol{P}^{*}\right)=0
$$

The bisection method is adopted to solve (26), when $F\left(\eta^{*}\right)=0, \eta^{*}$ is the optimal EE. Assume that the boundary of EE value are $\eta_{l}=0$ and $\eta_{u}=\psi$, respectively. $\psi$ is a large positive number, and we can guarantee the value of objective function (25) is less than zero. Firstly, we get the arithmetic mean of $\eta_{l}$ and $\eta_{u}$, then obtain the optimal power distribution $\boldsymbol{P}^{*}$ though resolving the optimization problem $\mathbf{P 5}$, where $\eta=\frac{\eta_{l}+\eta_{u}}{2}$, EE value $\eta_{i}$ can be calculated by equation (5) based on $p^{*}$, where $i$ is 
the iteration index. If $\eta_{i}>\eta, \eta_{l}=\eta$, otherwise, $\eta_{u}=\eta$. Repeat the above processes, until $\left|\eta_{u}-\eta_{l}\right| \leq v$, where $v$ is a very small positive step. The EE-based optimal power allocation algorithm is outlined in Table 4.

Table 4. EE based Optimal Power Allocation Alogrithm

\section{EE based Optimal Power Allocation Alogrithm}

1: Initialization: $i, v, \eta_{l}, \eta_{u}$ and $I_{m}$

2: Repeat

3: $\quad$ let $\eta=\frac{\eta_{l}+\eta_{u}}{2}$

4: $\quad$ Solve $\mathbf{P 5}$ to obtain $\boldsymbol{P}^{*}$

5: Calculate $\eta_{i}=\frac{R_{\text {tot }}\left(\boldsymbol{P}^{*}\right)}{P_{\text {tot }}\left(\boldsymbol{P}^{*}\right)}$

6: if $\eta_{i}>\eta$ then

7: $\quad \eta_{l}=\eta$

8: else

9: $\quad \eta_{u}=\eta$

10: end if

11: Until $\left|\eta_{u}-\eta_{l}\right| \leq v$ or $i \geq I_{m}$

12: Output: the maximum EE

\section{Simulation Results and Performance Evaluations}

In this section, the amount of simulation results is presented to qualify the performance of the proposed energy efficiency resource allocation algorithms in two DAS scenarios. In the simulations, the wireless channel condition is based on descriptions in the system model, and in both the spare and the dense scenarios. Table 5 shows the main simulation parameters in the simulation.

Table 5. Simulation parameters

\begin{tabular}{|c|c|}
\hline Parameters & Values \\
\hline \hline Cell radius & \\
\hline Path loss exponent & \\
\hline Lognormal shadowing & \\
\hline Drain efficiency & \\
\hline Circuit power & \\
\hline Backhaul power & \\
\hline Subcarrier bandwidth & \\
\hline
\end{tabular}




\subsection{In the sparse DAS}

In sparse DAS scenario, we analyze EE performance of the proposed JDSA algorithm and compare the performance of different algorithms versus subcarriers number, users number for different parameters.

Fig. 2 and Fig. 3 describes the convergence of the proposed JDSA algorithm with different parameters. From two figures, we could see that as the number of iterations increases, the proposed procedure tends to a certain value finally. In Fig. 2, we compare the value of EE for numbers of users and DAUs with a given subcarrier number $\mathrm{N}$. We find that EE is an increasing function of the number of users, and decreasing function of the number of DAUs. Specifically, when and, the maximum EE is achieved, this is because the fewer number of DAUs consumes the less power, and the larger number of users provides the larger multi-user diversity gain compared with other cases. EE with is higher than , this is due to power consumption of DAUs has lower effect on EE than multi-user diversity gain.

Fig. 3 illustrates EE of DASs for numbers of users and subcarriers with a given DAU number. It can be seen that EE increases with the increasing number of subcarriers. This is because the more subcarriers can bring higher data rate, and then improve EE. Specifically, EE of DASs is improved nearly 50\%, when $\mathrm{N}$ increases from 8 to 16.

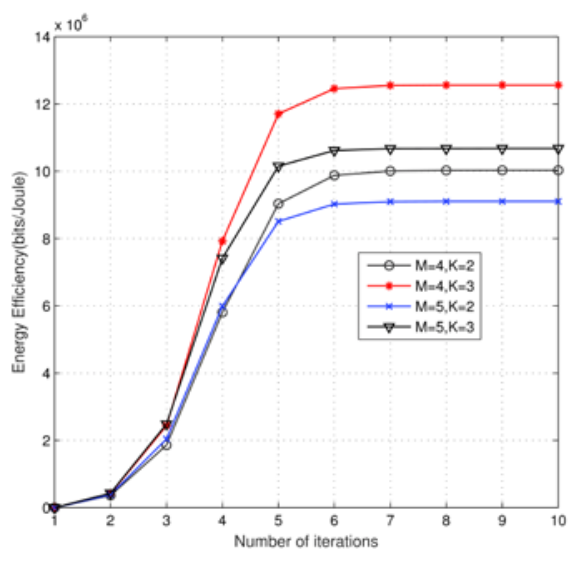

Fig. 2. EE versus iterations number with different $M, K$

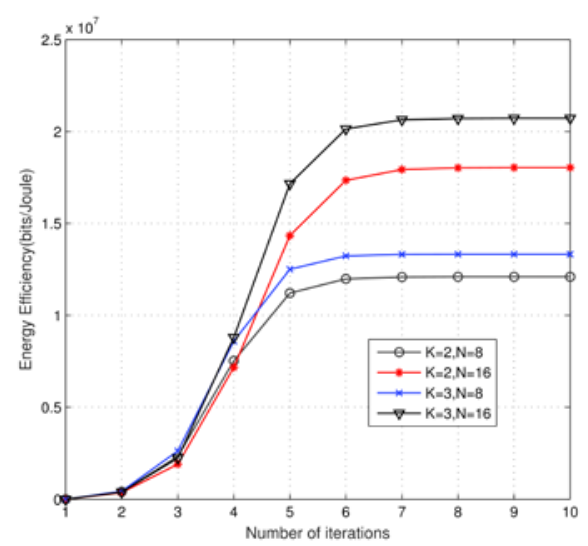

Fig. 3. EE versus iterations number with different $K, N$ 


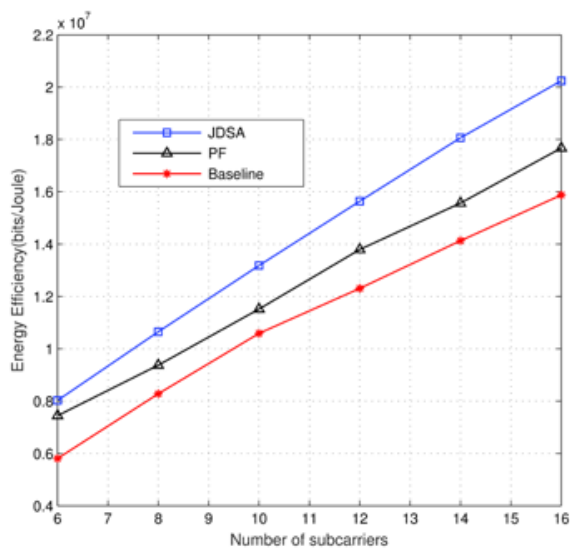

Fig. 4. EE versus subcarriers number for different algorithms

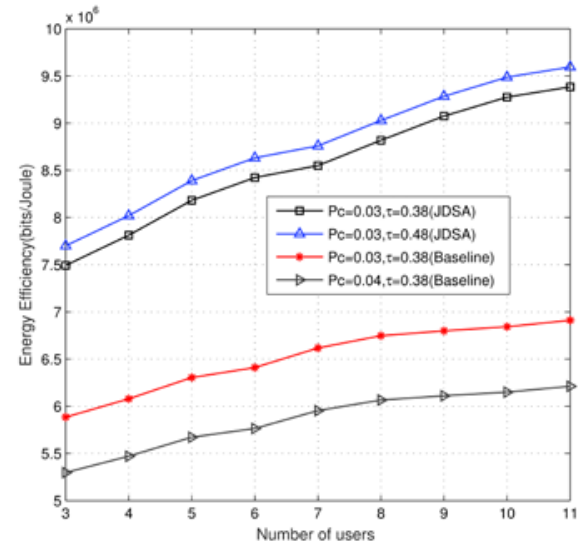

Fig. 5. EE versus users number with different $p_{c}$

Fig. 4 compares the EE of the system versus subcarriers number for three algorithms. $\mathrm{PF}$ algorithm is the proportional fairness algorithm, which allocates the subcarrier with the best channel condition to the user with the minimum transmission rate. Baseline algorithm allocates the subcarriers and DAUs randomly. From Fig. 4, as the number of subcarriers increases, EE of the system in three algorithms increases. Obviously, the proposed JDSA algorithm has better performance than the PF and the Baseline algorithm. Specifically, the EE gain of the JDSA algorithm and the PF, the Baseline algorithm is an increasing function of the number of subcarriers.

Fig. 5 shows the relationship between EE and UEs with different parameters. As the number of UEs grows, all the curves in Fig. 5 show an upward trend. we compare the proposed JDSA algorithm with the Baseline algorithm. It is shown that the JDSA algorithm could achieve higher EE than the baseline algorithm. Furthermore, we evaluate the influence of circuit power and power amplifier efficiency to total EE. From the simulation results, it is clear that higher results in lower EE, meanwhile larger could be benefit for EE.

\subsection{In the dense DAS scenario}

In the dense DAS scenario, we assume that the maximum transmission power of all the UEs is equal, and the minimum data rate requirement is. We analyze the convergence behavior of the proposed algorithm, the effect of the virtual cell size, the number of total DAUs, and the tradeoff between EE and spectrum efficiency (SE) for different algorithms. 


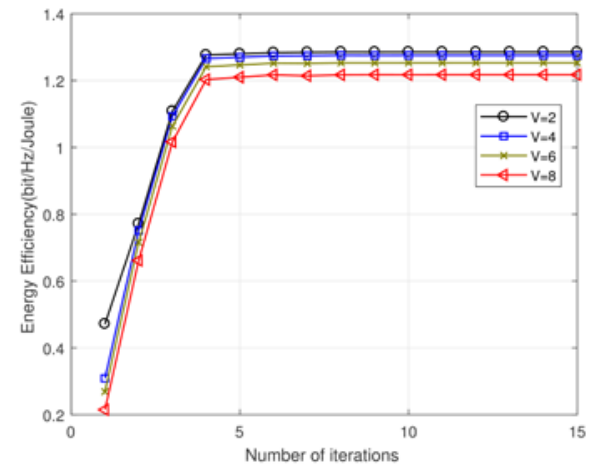

Fig. 6. EE versus iterations number with different $V$

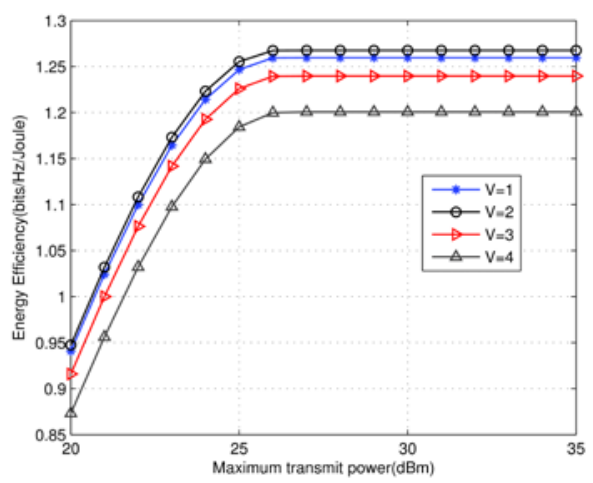

Fig. 7. EE versus maximum transmission power with different $V$

In Fig. 6, we show the convergence of the proposed algorithm. It can be seen that the proposed algorithm converges in few iterations. Meanwhile, it can be observed that EE decreases as the increasing size of virtual cell, this is because larger virtual cell consumes more power.

In Fig. 7, we show EE of DASs versus maximum transmission power with different size of the virtual cell. EE increases as increases when, and keep steady after that. In addition, the maximum EE is achieved when, since users can attain more macro diversity and the system does not consume more power. Furthermore, EE is higher at than. This is because the gain from transmission data rate is higher than the loss from the power consumption. However, when, more power is dissipated, leads to a lower EE.

Fig. 8 compares EE versus maximum transmit power with different when. In Fig. 8, EE increases with increases, and EE keeps stable when. When, the maximum EE is approximately $11 \%$ higher than, and EE is higher at than. This is because the difference of the average distance between users and DAUs decreases when the number of DAUs increases.

In Fig. 9, we provide performance comparisons for different circuit power and power amplifier efficiency with and. We can see that the increasing of the circuit power leads to the decreasing of the EE, and enhance the power amplifier efficiency can improve EE. 


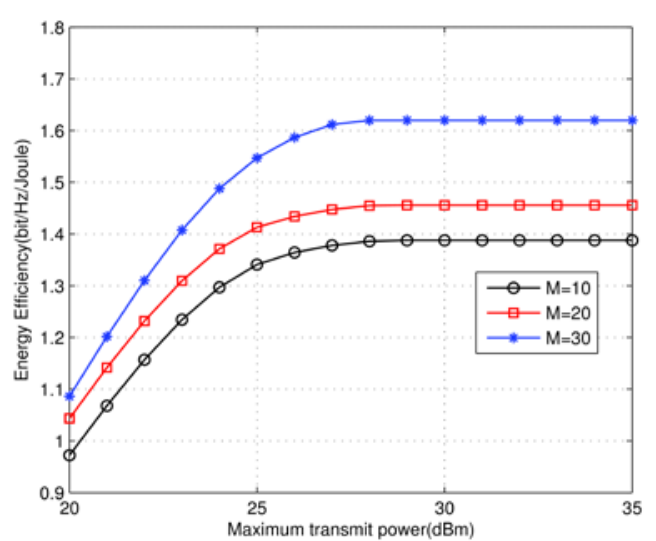

Fig. 8. EE versus maximum transmission power with different $M$

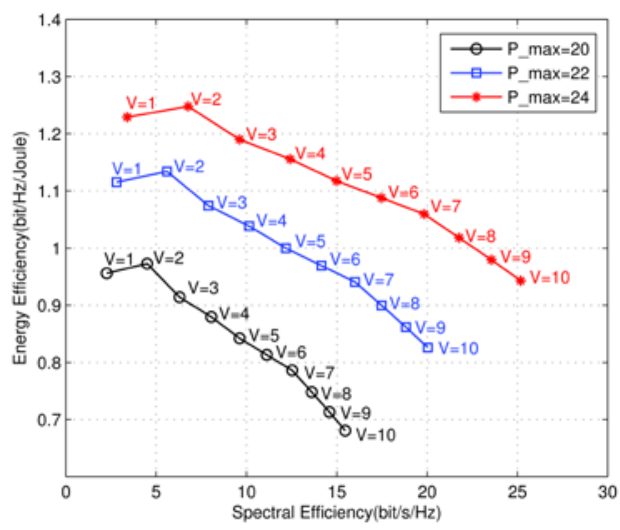

Fig. 10. EE versus $S E$ with different $p^{\max }$

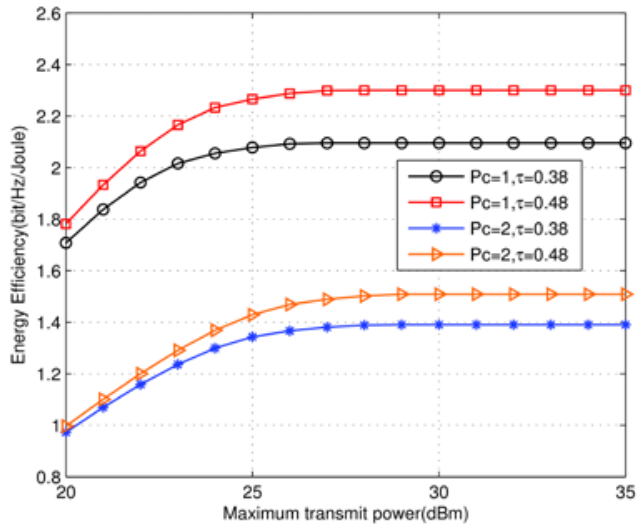

Fig. 9. EE versus maximum transmission power with different $P_{c}, \tau$

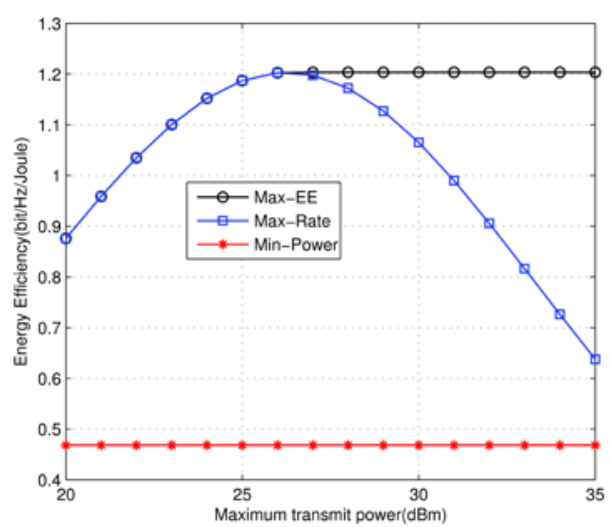

Fig. 11. EE versus maximum transmission power

Fig. 10 is the relationship between EE and SE for different . In Fig. 10, EE decrease as SE increases. Since more power is consumed with increasing SE, the proper number of DAUs can not only satisfy SE requirement but also improve EE. Moreover, EE increases as increases.

Fig. 11 is the comparation of various methods according to with , the Max-Rate scheme under transmit power constraint for all DAUs, the Min-Power scheme which minimizes the total power consumption under the minimum SE requirement and the proposed Max-EE scheme.

Based on the results, we can see that EE of the Min-Power scheme is a constant and EE of Max-Rate scheme increases when, then decrease after that. This is because the Max-Rate scheme aims to maximize SE, which will consume more power. For the 
proposed Max-EE scheme, EE increases as increases when, then keep stable after that. The optimal EE is obtained at, and the system will be bounded by other constraints since then, thus increase can not improve EE. In addition, when EE of the Max-EE is the same as the Max-rate scheme. Notice that, the transmission power allocated to DAU is the same for both schemes.

\section{Conclusion}

In this paper, we studied energy efficiency resource allocation in a downlink multiuser DAS with different DAU density. A joint DAU selection with subcarrier allocation algorithm and optimal power allocation algorithm were proposed to maxmize EE under the constrains of transmit power and DAU selection rules. Particularly, co-channel interference was considerd in the dense DAS scenario since users could access the same channel. Due to the nonconvex property of the original problem, we introduced interference upper bound and fractional programming theory to solve it. Through the algorithm simulation diagrams, we can vertify the efficiency of the proposed algorithm.

\section{References}

[1] N. Saquib, E. Hossain, L. B. Le and D. I. Kim, "Interference management in OFDMA femtocell networks: issues and approaches," IEEE Wireless Communications, vol. 19, no. 3, pp. 86-95, June 2012. Article (CrossRef Link)

[2] L. Dai, "A Comparative Study on Uplink Sum Capacity with Co-Located and Distributed Antennas," IEEE Journal on Selected Areas in Communications, vol. 29, no. 6, pp. 1200-1213, June 2011. Article (CrossRef Link)

[3] Wonil Roh and A. Paulraj, "Outage performance of the distributed antenna systems in a composite fading channel," in Proc. of IEEE 56th Vehicular Technology Conference, vol.3, pp. 1520-1524, 2002. Article (CrossRef Link)

[4] C. He, B. Sheng, P. Zhu, X. You and G. Y. Li, "Energy- and Spectral-Efficiency Tradeoff for Distributed Antenna Systems with Proportional Fairness," IEEE Journal on Selected Areas in Communications, vol. 31, no. 5, pp. 894-902, May 2013. Article (CrossRef Link)

[5] X. Chen, X. Xu and X. Tao, "Energy Efficient Power Allocation in Generalized Distributed Antenna System," IEEE Communications Letters, vol. 16, no. 7, pp. 1022-1025, July 2012. Article (CrossRef Link)

[6] X. Li, X. Ge, X. Wang, J. Cheng and V. C. M. Leung, "Energy Efficiency Optimization: Joint Antenna-Subcarrier-Power Allocation in OFDM-DASs," IEEE Transactions on Wireless Communications, vol. 15, no. 11, pp. 7470-7483, Nov. 2016. Article (CrossRef Link)

[7] C. He, G. Y. Li, F. C. Zheng and X. You, "Energy-Efficient Resource Allocation in OFDM Systems With Distributed Antennas," IEEE Transactions on Vehicular Technology, vol. 63, no. 3, pp. 1223-1231, March 2014. Article (CrossRef Link)

[8] Xiaoge Huang, Baltasar Beferull-Lozano and Carmen Botella, "Quasi-Nash Equilibria for Non-Convex Distributed Power Allocation Games in Cognitive Radios," IEEE Transactions on Wireless Communications, vol. 12, no. 7, pp. 3326-3337, July 2013.

Article (CrossRef Link) 
[9] Weiye Xu, Min Lin, Yu Yang and X. Yu, "Adaptive energy-efficient power allocation for DAS with imperfect channel state information and antenna selection," China Communications, vol. 13, no. 7, pp. 127-134, July 2016. Article (CrossRef Link)

[10] B. Hu, Q. Sun, J. Wang and C. Xu, "Joint power allocation and antenna selection for energy-efficient OFDM D-MIMO systems," in Proc. of 2014 Sixth International Conference on Wireless Communications and Signal Processing (WCSP), Hefei, pp. 1-5, 2014. Article (CrossRef Link)

[11] Y. Li, M. Sheng, X. Wang, Y. Shi and Y. Zhang, "Globally optimal antenna selection and power allocation for energy efficiency maximization in downlink distributed antenna systems," in Proc. of 2014 IEEE Global Communications Conference, Austin, TX, pp. 3856-3861, 2014. Article (CrossRef Link)

[12] Fangmin $\mathrm{Xu}$ and $\mathrm{Y} . \mathrm{Hu}$, "Antenna selection and power allocation algorithm in distributed antenna systems," in Proc. of 2015 IEEE 16th International Conference on Communication Technology (ICCT), Hangzhou, pp. 540-543, 2015.

[13] M. Ndong and T. Fujii, "Joint antenna selection and power allocation for distributed-stbc cognitive small cell networks," in Proc. of 2014 International Symposium on Wireless Personal Multimedia Communications (WPMC), Sydney, NSW, pp. 439-444, 2014. Article (CrossRef Link)

[14] J. Li, J. Yan, L. Zhao and Q. Dong, "Antenna selection and transmit beamforming optimisation with partial channel state information in distributed antenna systems," IET Communications, vol. 8, no. 13, pp. 2272-2280, September 5 2014. Article (CrossRef Link)

[15] Xiaoge Huang, Weipeng Dai, Zhifang Zhang, Qiong Huang, Qianbin Chen, "Energy-Efficient Resource Allocation in Distributed Antenna Systems," in Proc. of Chinacom, 2016 CHINACOM 11th EAI International Conference on Communications and Networking, China, September 2016.

[16] G. Xu, A. Liu, W. Jiang, H. Xiang and W. Luo, "Joint user scheduling and antenna selection in distributed massive MIMO systems with limited backhaul capacity," China Communications, vol. 11, no. 5, pp. 17-30, May 2014. Article (CrossRef Link)

[17] Werner Dinkelbach, "On nonlinear fractional programming," Management Science, vol. 13, no. 7, pp. 492-498, March 1 1967. Article (CrossRef Link)

[18] Z. Q. Luo and S. Zhang, "Dynamic Spectrum Management: Complexity and Duality," IEEE Journal of Selected Topics in Signal Processing, vol. 2, no. 1, pp. 57-73, Feb. 2008. Article (CrossRef Link) 

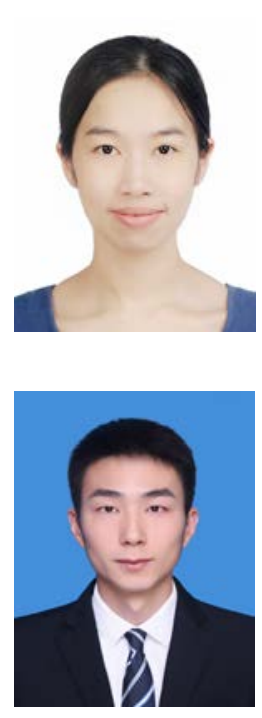

Dongyu Zhang received the B.S. degree in Electronic and Information Engineering from Zhengzhou University of Light Industry, Henan, China, in 2016. He is currently working toward the M.S. degree in Electronics and Communication Engineering with Wireless Transmission Laboratory, Chongqing University of Posts and Telecommunications, Chongqing, China. His main research interests are cognitive radio network, wireless communication, and small cell network.

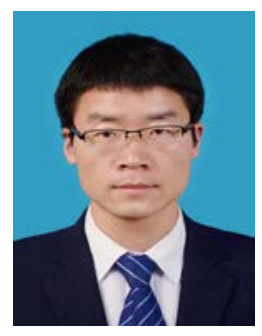

Weipeng Dai received the B.S. degree in Electronic Information Science and Technology from Chongqing University of Technology in 2014. And in 2017, he received the M.S. degree in Electronics and Communication Engineering with Wireless Transmission Laboratory, Chongqing University of Posts and Telecommunications, Chongqing, China. His main research is energy efficiency resource allocation in distributed antenna systems.

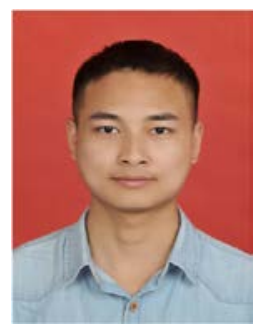

She Tang received the B.S. degree from Wuhan polytechnic university, Hubei, China, in 2016. He is working toward the M.S. degree in information and Communication Engineering with Wireless Transmission Laboratory, Chongqing University of Posts and Telecommunications, Chongqing, China. His main research interest is energy saving in small cell network. 